\title{
REFORMAS DEL CONGRESO Y REPRESENTACIÓN POLÍTICA EN VENEZUELA
}

\author{
Michael R. Kulisheck*
}

La democracia venezolana se encuentra en un período de transición entre el régimen del Pacto de Punto Fijo y el período de Hugo Chávez. La fórmula política del Pacto, definido por partidos fuertes y acuerdos por parte de las élites, empezó a fallar en los 80 y fracasó totalmente en los 90 . Este fracaso se debió al peso de una política cada vez más cerra$\mathrm{da}$, una dependencia excesiva en las ganancias petroleras para promover el desarrollo económico, y una congelación de los vínculos de los intereses sociales y sus representantes en el gobierno'.

Hugo Chávez ganó las elecciones presidenciales con un plan que llamó la atención a las imperfecciones de representación en el sistema puntofijista. Ahora Chávez y otros actores "nuevos" dominan la política venezolana. Cuando la Asamblea Constituyente reescriba la Constitución, los actores y las reglas-del-juego del régimen del Pacto de Punto Fijo se convertirán en parte del pasado histórico de la nación venezolana.

La elección de Chávez puede ser entendida solamente teniendo en cuenta el limitado impacto de los cambios políticos e institucionales. En los años 90, los reformistas anunciaron nuevas leyes electorales y la derrota del bipardismo como estrategias para revitalizar la representación en el sistema político actual. De acuerdo con la nueva ley electoral mixta, una mitad de la Cámara de Diputados era elegida en nuevos circuitos uninominales en 1993, mientras que la otra mitad continuaba siendo elegida por listas partidistas cerradas. Aunque no fue una reacción a la nueva ley electoral, cinco partidos ganaron representación significativa en 1993 por primera vez en veinticinco años. Las estructuras de los partidos nuevos -Convergencia, La Causa-R, y MAS (Movimiento al Socialismo)- son más decentralizadas y menos verticales que las de los partidos de Acción Democrática y COPEI que definieron el bipardismo por más de dos décadas.

Los reformistas predijeron que las carreras políticas de los diputados de los nuevos circuitos uninominales y de los partidos descentralizados dependerían menos de las maquinarias de los partidos y de las élites que las de quienes pertenecían a listas partidistas y partidos centralizados. Se esperaba que esta independencia de las maquinarias de los partidos animara a los diputados a consolidar los vínculos con los grupos de interés emergentes a nivel estatal y local.
Estos intereses habían sido excluidos de los procesos formales políticos y de los marcos de los partidos dominantes desde su fundación a finales de los 70 y en los 80 . La ampliación de la representación de la política formal se vio como una manera de reestablecer el equilibrio político en Venezuela y de reducir el apoyo a opciones políticas radicales como la del golpista Hugo Chávez. Las expectativas de los reformistas fueron erróneas. A pesar de la reforma electoral y del colapso del sistema bipartidista, Hugo Chávez ganó las elecciones de 1998.

El presente artículo analiza el impacto de las estructuras partidistas y las leyes electorales en la representación en la Cámara de Diputados (1994-1999) para entender la popularidad de Chávez y su plataforma para el cambio radical. En el artículo se usarán datos de la encuesta realizada por el autor a diputados venezolanos (Kulisheck 1997). Dicha encuesta fue llevada a cabo durante los meses de abril, mayo y junio de 1997 ( $N=65 ; 31,4 \%$ de la Cámara de Diputados), y contenía 42 preguntas con respuestas cerradas sobre patrones de representación y actividades legislativas de los diputados.

De manera inesperada, como resultado de esta encuesta, se descubrió que los diputados de los circuitos uninominales y de los partidos descentralizados no cultivan vínculos con los intereses emergentes, sino que se alían con los intereses tradicionales como la patronal y los sindicatos. De todas maneras, en vez de promover la representación de los intereses emergentes que, a nivel estatal y local, habían estado alienados de la política formal, la nueva ley electoral y las estructuras partidistas reforzaron los patrones de representación del régimen de Punto Fijo. Chávez se aprovechó de la opinión y el descontento del pueblo sobre los fallos de la estrategia reformista para renovar la política venezolana.

Este artículo está dividido en cuatro partes. La primera revisa los orígenes de la representación partidista en Venezuela, cómo funcionó y decayó en los 80 . La segunda parte

* Doctorado en Ciencias Políticas de la Universidad de Pittsburgh Instructor adjunto de la Universidad del Estado de Colorado. EE.UU Agradezco a María del Mar López-Cabrales sus sugerencias y al Centro de Estudios Latinoamericanos de la Universidad de Pittsburgh y al Programa de Fulbright IIE por su apoyo financiero durante esta investigación.

1. Véase Alvarez (1996a); Canache y Kulisheck (1998); Goodman et al. (1995); Karl (1997); McCoy et al. (1995); Urbaneja (1995). 
analiza las respuestas sociales ante el fracaso de la representación partidista y, en consecuencia, las organizaciones de la sociedad civil y de otras alternativas políticas más radicales. Esta parte también hace una revisión de la estrategia reformista para estabilizar la política en Venezuela a través de la adopción de nuevas leyes electorales y de la desaparición del sistema bipartidista. La parte tercera examina los efectos de la estructura partidista y de las leyes electorales en los patrones de representación en la Cámara de Diputados de 19941999. Finalmente, la cuarta y última parte analiza la campaña electoral como ejemplo del fracaso de las reformas electorales a la hora de mejorar la representación en la política formal.

\section{REPRESENTACION PARTIDISTA (1958-1980)}

El sistema político venezolano ha sido indentificado como "partidocracia" (Brewer-Carías 1988; Coppedge 1994) debido al control que ejercen los partidos en la esfera de los procesos, la representación y las decisiones políticos. Esta relación institucionalizada y simbiótica entre partidos e intereses organizados funcionó eficazmente durante los primeros quince años de la democracia en Venezuela. Para los partidos, los vínculos estrechos con las organizaciones sociales fueron la causa de la movilización de los votantes. Para las organizaciones, la afiliación a distintos partidos tuvo como resultado una conexión bastante eficaz con el gobierno y la burocracia (Coppedge 1994: 27). Sin embargo, el modelo de representación partidocrático dificultó la situación a nivel local y estatal y los intereses emergentes en la sociedad civil no pudieron entrar en la arena política cuando se formaron a finales de los 70 y 80 .

Durante la transición democrática en 1958, el Pacto de Punto Fijo estableció las condiciones para que los partidos dominaran la representación del sistema político emergente. En el Pacto, las élites de los partidos políticos más importantes, los sindicatos y la patronal dejaron a un lado sus diferencias políticas e ideológicas para entablar acuerdos formales sobre instituciones políticas básicas y las reglas-del-juego (Karl 1986; Levine 1973; Rey 1972). El Pacto de Punto Fijo no creó ni reglas ni instituciones específicas, sino que estableció los patrones generales para tomar decisiones de manera conciliatoria. Las élites acordaron que los partidos serían los mediadores principales en la arena política y que los temas controvertidos se discutirían en privado entre los firmantes del Pacto en vez de hacerlo en debates públicos en el Congreso (Levine 1978: 93-94).

Los partidos venezolanos pudieron cumplir las promesas que hicieron en el Pacto de Punto Fijo gracias al poder y organización que tenían en la sociedad. La influencia de los partidos venezolanos surgió de sus estructuras institucionales y de las circunstancias históricas fundacionales de cada partido en los 40. Debido a que las organizaciones autónomas venezolanas en su totalidad fueron eliminadas sistemáticamente en la dictadura de Gómez (1908-1936), los partidos se formaron a partir del vacío político de finales de los 30 y 40. Como resultado, muchos de los grupos e intereses que se habían organizado de manera autónoma, se prepararon dentro del marco de los partidos políticos (Levine 1978:
86-87). Por ejemplo, algunos activistas de la Acción Democrática y otros partidos fueron instrumentos esenciales para la movilización, entre otras cosas, de los sindicatos, grupos de campesinos, asociaciones del cuerpo docente, y organizaciones de estudiantes y profesionales. Después, cuando los grupos nuevos expresaban demandas políticas eran integrados en las estructuras del partido y casi se convirtían en partes corporativas de los partidos más importantes (Salamanca 1995: 200).

En este contexto, Rómulo Betancourt, fundador de Acción Democrática, junto con otros activistas trabajaron para construir un partido que estuviera presente en todos los niveles de la vida nacional y local, y fuera capaz de integrar muchos intereses y grupos en la organización (Kornblith y Levine 1995: 41). El activismo en el partido será constante y la democracia será practicada en elecciones frecuentes para escoger representantes a comisiones estatales, regionales y nacionales. Betancourt vio a Acción Democrática como una extensión del pueblo venezolano (Martz 1992: 111). El éxito electoral de Acción Democrática sirvió de ejemplo a otra gente, especialmente a Rafael Caldera de COPEI que copió esta estrategia organizativa. Como resultado, los partidos con éxito en Venezuela han sido heterogéneos socialmente, y han combinado una representación de distintos grupos combinando una centralización y un liderazgo fuertes (Kornblith, Levine 1995: 41).

La organización vertical de Acción Democrática y COPEI promovió la representación política en Venezuela al unir el pueblo con la élite, el interior con la capital, y los grupos de interés con el gobierno y la burocracia. Las oficinas de los partidos situadas en los pueblos más pequeños eran puntos de contacto entre los ciudadanos, los partidos y el gobierno. La gente llevaba directamente sus quejas y peticiones a los organizadores locales del partido. Los líderes locales de los partidos pasaban dichas peticiones a la organización estatal del partido y, si estas quejas tenían sentido, llegaban a Caracas donde los miembros del partido en el gobierno y la burocracia tomaban acción (Karl 1997: 107).

Debido a su influencia institucional en los procesos políticos y su poder en la sociedad, los partidos han funcionado como mediadores de representación política en el congreso (Rey 1972: 205). Acción Democrática y COPEI llegaron a ocupar un 75\% de escaños en el congreso de 1973 a 1993. Por ello, los intereses independientes tuvieron que negociar con Acción Democrática y COPEI si querían influir en las decisiones políticas. Los procesos rígidos de disciplina crearon incentivos para que los legisladores de los partidos más importantes pusieran atención en los intereses tradicionales con vínculos en dichos partidos. Una vez que se decidía una línea partidista, los legisladores que abiertamente estaban en contra sufrían castigos rápidos por parte de los tribunales disciplinarios de sus partidos (Rey 1972: 202).

Las estructuras centralizadas partidistas comenzaron a arriesgar, en vez de promover, la representación que comenzó en los años 70 (Martz 1998: 67). En lugar de usar la influencia política para representar las preocupaciones de sus miembros, la élite partidista consolidó el poder personal y sus posiciones de liderazgo (Martz 1992: 102). El cambio de liderazgo fue algo casi imposible y los lazos entre la 
élite caraqueña y los miembros regulares de los partidos se debilitaron. Para Acción Democrática y COPEI, la representación se convirtió en algo importante sólo para conseguir más votos. Debido a que los partidos ejercían tal control en la arena política, la ruptura de los vínculos entre el pueblo y la élite partidista minó la representación significativa en Venezuela. Incluso las organizaciones con vínculos en partidos fueron conscientes de que sus intereses no estaban tan bien representados como en el pasado ${ }^{2}$.

La representación fue complicada en los 80 , porque las expectativas de lo que la gente podía conseguir a través de la política estaban fundamentadas en la época del boom petrolero de los 70. La ganancia del petróleo creó la impresión de una representación eficaz durante este período. "El petróleo proporcionó las ganancias fiscales necesarias para que las administraciones democráticas mantuvieran la ambigua y cara situación de fomentar el crecimiento del sector privado, a la vez que favorecía a la clase media y trabajadora" (Karl 1986: 215). Los beneficios del boom petrolero del 73 y 79 crearon las fuentes necesarias para que ambos partidos (el gobernante y los opositores) satisfacieran las demandas del electorado y fueron percibidos, a la vez, responsables por los intereses, incluso hasta cuando los vínculos entre élite y pueblo en el sistema partidista estaban fracasando (Karl 1995: 36). La caída de los precios del petróleo al principio de los 80 minó la posibilidad de mantener un sistema de representación financiado por las ganancias del petróleo. Sin estos recursos económicos, los partidos enfrentaron grandes dificultades cuando intentaban acomodar las peticiones de sus aliados políticos.

El apoyo público a las instituciones y procesos políticos disminuyó a la vez que los partidos no confrontaron las preocupaciones de la mayoría de los venezolanos. De 1973 a 1992 las actitudes de la gente hacia el gobierno empeoraron y el índice de la miseria económica aumentó (la suma de las cifras de desempleo e inflación). En 1973, el índice de la miseria era del $17 \%$ y el $37 \%$ de los venezolanos tenía una actitud favorable hacia el gobierno. En 1983 el índice de la miseria había subido al $28 \%$ y el porcentaje de las actitudes favorables hacia el gobierno bajó al 16. En 1992 el índice de la miseria era del $41 \%$ y sólo $13 \%$ de los ciudadanos expresó una actitud favorable hacia el gobierno (Templeton 1995: 84). Los índices favorables hacia el congreso y los partidos políticos eran del 12,4 y 6,1\% respectivamente en 1992 (Alvarez 1996b: 151). Los venezolanos se habían dado cuenta de la ineficacia de la representación partidista.

\section{RESPUESTAS POLÍTICAS A LA CAÍDA DE LA REPRESENTACIÓN PARTIDISTA}

Los venezolanos respondieron a los problemas de representación de tres maneras. La primera consistió en que los ciudadanos formaron organizaciones en la sociedad civil, en concreto a nivel local, para enfrentar las preocupaciones que no habían encontrado representación en la arena política formal. A la vez, se crearon nuevas alternativas políticas más radicales que defendían la idea de que la representación no mejoraría hasta que los acuerdos y actores políticos del sistema de Punto Fijo fueran reemplazados. La segunda se basó en que casi una mayoría de ciudadanos no votaron por Acción Democrática y COPEI en las elecciones legislativas de 1993 rompiendo, de esta manera, con el bipartidismo. Y la tercera respuesta se dio cuando la élite política adoptó una nueva ley electoral mixta para la Cámara de Diputados para ampliar la representación legislativa. Si las organizaciones de la sociedad civil se hubieran beneficiado de los cambios del sistema partidista y de la reforma electoral para mejorar sus contactos con sus representantes, el programa de cambio político radical de Hugo Chávez no habría encontrado el masivo apoyo de la gente venezolana en las elecciones de 1998.

A continuación se examinará el surgimiento de grupos en la sociedad civil y sus estrategias para influir en los procesos políticos a través de actividades en la política informal y sus esfuerzos para reformar el Estado; y se explorará el fracaso del sistema bipartidista en las elecciones de 1993 como una respuesta del pueblo ante los fallos del liderazgo del sistema puntofijista: Acción Democrática y COPEI. También se revisarán las estructuras organizativas de los tres partidos (Convergencia, MAS, La Causa-R que ganaron una representación significativa por primera vez en 1993. Finalmente, se analizará cómo la adopción de la ley electoral mixta de la Cámara de Diputados se justifica porque mejora la representación y restablece la legitimidad democrática.

\section{La aparición de las organizaciones de la sociedad civil y de las alternativas políticas radicales}

El crecimiento de las organizaciones de la sociedad civil se correspondió con el aumento de los sentimientos de alienación que los ciudadanos tenían con respecto a los representantes en los partidos más importantes y en el gobierno. Los nuevos grupos e intereses encontraron las reglas-deljuego ya existentes que favorecían los intereses tradicionales vinculados a Acción Democrática y COPEI (Levine y Crisp 1995: 240; Salamanca 1995: 203). Los objetivos de la mayoría de las organizaciones de la sociedad civil estaban condicionados por las necesidades de sus miembros, y sus estrategias adaptadas a la naturaleza cerrada del sistema de Punto Fijo. En concreto, los grupos de la sociedad civil se formaron 1) para confrontar asuntos que habían sido ignorados en la arena política formal dominada por Acción Democrática y COPEI y 2) para pedir reformas que ampliaran la participación en los procesos políticos e hicieran la representación más eficaz.

La arena política informal ha sido un espacio importante en la que los grupos han producido bienes y servicios inmediatos y tangibles para sus miembros y sus comunidades (Hochstetler 1997: 202). Muchas organizaciones de la sociedad civil habían trabajado en la política informal para confrontar las necesidades sociales que no habían sido cubiertas por los partidos y el gobierno de la nación. El tipo de servicios y bienes que las organizaciones de la sociedad civil

2. El sindicato insurgente, asociado con La Causa-R, se movilizó en los 70 y 80 porque los sindicatos controlados por Acción Democrática (como la Confederación de Trabajadores Venezolanos-CTV) no estaban promocionando eficazmente los intereses de los trabajadores (Hellinger 1996). 
provee para sus ciudadanos es diverso. Por ejemplo, el movimiento de los vecinos se formó en los suburbios de Caracas a finales de los 70 para luchar contra el crecimiento no planificado de la ciudad (Levine 1998: 200). Durante el segundo gobierno de Carlos Andrés Pérez, algunos de estos grupos de vecinos distribuyeron leche subvencionada por el Estado en barrios pobres (Gómez Calcaño 1998: 181). Las cooperativas se organizaron para proteger a grupos pequeños de habitantes de los precios altos y para que el transporte, el capital y los créditos fueran más accesibles a las clases medias y bajas venezolanas (Crisp y Levine 1998: 41). CESAP (Centro al Servicio de la Acción Popular) creó programas de formación y cursillos para grupos locales y estatales, así como individuales programas de alfabetización y guarderías (Levine y Crisp 1995: 241). Organizaciones como las Ferias del Consumo Popular se formaron para promover la vida en comunas en zonas mayoritariamente pobres y así solucionar problemas como la alimentación, el desempleo y la salud (Salamanca 1995: 207).

Las organizaciones de la sociedad civil han usado la política simbólica para redefinir el debate político fuera de los partidos más importantes. AMIGRANSA (Asociación de Amigos de Defensa de la Gran Sabana) y otros miembros del movimiento ambiental venezolano, por ejemplo, han usado la política simbólica para alertar a la sociedad de la destrucción de partes del Parque Nacional de Canaima. Estos grupos han sido significativos porque sus temas y valores han creado nuevos asuntos políticos y, a la vez, el uso que hacen de los medios de comunicación ha ampliado el entendimiento y conceptualización masivos de sus intereses existentes (García-Guardilla 1992: 159-161). El movimiento de los vecinos también ha usado los medios de comunicación para promover su agenda. La Escuela de Vecinos de Venezuela usa los programas de la radio y un show televisivo diario para extender sus noticias sobre sus grupos y proyectos de la comunidad (Levine 1998: 201).

Para mejorar la representación en la política local, estatal y nacional muchas organizaciones de la sociedad civil se han movilizado para transformar el sistema político. En los 80, dichas transformaciones en Venezuela fueron más reformistas que revolucionarias, y consistieron en el aumento de la responsabilidad del gobierno y en el desarrollo del poder más allá de las instituciones y los actores a nivel nacional (Hochstetler 1997: 199). Las organizaciones de la sociedad civil en Venezuela han transformado el Estado incorporando en la agenda de la nación las reformas necesarias, y se han basado en una movilización en la política informal para promover esta agenda reformista.

Ejemplos de esfuerzos en las transformaciones estatales son las campañas para cambiar las leyes electorales y descentralizar las responsabilidades económicas y políticas del gobierno nacional en los estados y localidades (Crisp y Levine 1998: 43). En 1987, partes del movimiento de vecinos recogieron 140.000 firmas para pedir la reforma de las leyes básicas que regían los municipios (Ley Orgánica del Régimen Municipal-LORM). La reforma del LORM de 1988 y la aprobación de la Ley de Elección Directa de los Gobernadores de Estado dictaminaron la elección directa de los gobernadores y alcaldes, la creación de consejos locales y la posibilidad de "recall" para políticos locales y estatales (Levine 1998: 201). Estas reformas institucionales crearon espacios adicionales en los que los grupos, organizaciones y movimientos nuevos podían participar en política y los políticos podían forjarse carreras (Guerón y Manchisi 1996; Kornblith y Levine 1995; Kulisheck y Canache 1998). La organización de la sociedad civil "Queremos Elegir" se formó en 1991 para promover reformas electorales adicionales y su primer objetivo fue extender el uso de circuitos uninominales para elegir los escaños legislativos. Este grupo ha sostenido que los incentivos electorales unidos a las reglas de circuitos uninominales animan a los legisladores a estar más atentos a los intereses emergentes a nivel local y estatal (Gómez Calcaño 1998: 174).

La clase dirigente aceptó estas reformas relativamente moderadas a finales de los 80 para minar otras demandas más radicales. Los disturbios de 1989 habían amenazado a gran parte de la élite venezolana y habían dejado claro las consecuencias potencialmente violentas de la representación ineficaz en el sistema político. Como consecuencia del intento de golpe de estado de 1992, los políticos comenzaron a debatir algunos elementos del programa de reformas que las organizaciones de la sociedad civil habían desarrollado en 1980. Este segundo turno de discusión sobre la reforma fue indudablemente parte de una estrategia para no apoyar a alternativas políticas más radicales.

La alternativa política radical, que se personificó en Hugo Chávez, se centró en un principio en los mismos asuntos que preocupaban a las organizaciones de la sociedad civil. Por ejemplo, la crítica del sistema bipartidista como corrupto y no representativo, un sistema que llevaría a la formación del Movimiento Bolivariano 200 (MBR-200) en el ejército a mediados de los 80 . Los grupos de la sociedad civil trabajaron con las reglas-del-juego de Punto Fijo y el MBR-200, bajo el liderazgo del teniente coronel Hugo Chávez, dio un golpe de estado que estuvo a punto de triunfar en febrero de 1992. Chávez aceptó total responsabilidad del fracasado golpe de estado en un cominicado que dio en televisión, pero su crítica a Acción Democrática, COPEI, y la patronal resonó en los sectores de la clase media y baja y, rápidamente, se convirtió en una alternativa popular a los líderes partidistas tradicionales que eran percibidos como personas corruptas políticamente (Levine 1994).

Chávez fue perdonado por el nuevo presidente Rafael Caldera en 1994, y rápidamente convirtió a MBR-200 en un movimiento sociopolítico (López Maya 1998). Para protestar contra la corrupción del sistema, MBR-200 no tuvo candidatos en las elecciones locales y estatales de 1995 y animó a los venezolanos a abstenerse. Sin embargo, en 1997 Chávez decidió presentarse como candidato a las elecciones presidenciales, y para ello formó el Movimiento V República (MVR). Chávez promovió su candidatura enfatizando su desacuerdo con el sistema político controlado por Acción Democrática y COPEI; prometió convocar una Asamblea Constituyente para reescribir la Constitución e hizo vagas declaraciones en contra de la corrupción política y económica en la que Venezuela estaba embarcada debido al sistema bipartidista. En su campaña, Chávez echó la culpa de los problemas de la clase media y baja venezolana a la 
corrupción económica de la élite y de la patronal y a las decisiones injustas de los bancos internacionales como el Fondo Monetario Internacional y el Banco Mundial.

Los grupos de la sociedad civil y Hugo Chávez fueron ejemplos de actores políticos nuevos en los 80 y sus objetivos generales fueron parecidos. Los grupos de la sociedad civil intentaban reformar el sistema de Punto Fijo, aunque Chávez intentaba reemplazarlo. A mediados de los 90, las organizaciones de la sociedad civil comenzaron a ampliar su participación en la política formal y Hugo Chávez y sus vehículos políticos (MBR-200 y MVR) aceptaron las reglas-deljuego nacionales, pero sólo para cambiarlas. La opción radical o reformista dependía del éxito de los cambios políticos y de las reformas institucionales durante el período político de 1994-1999.

\section{El cambio del sistema partidista}

Las elecciones de 1993 marcan un cambio drástico en la formación partidista de la Cámara de Diputados ${ }^{3}$. Más de dos partidos ganaron representación significativa en el congreso por primera vez desde 1968. Los reformistas predijeron que la representación más amplia en la Cámara de Diputados crearía más oportunidades para que los intereses emergentes cultivaran un acceso independiente a la legislatura y minaran el apoyo del programa político chavista.

Acción Democrática y COPEI controlaron un promedio del 84\% de los escaños en el congreso desde 1973 hasta las elecciones de 1988 (Rey 1994: Apéndice 2). Por el contrario, cuando el nuevo congreso se inauguró en 1994 , Acción Democrática controlaba la delegación más numerosa, con un poco más del $25 \%$ de los escaños. Acción Democrática y COPEI juntos tenían el $54 \%$ de los escaños de la Cámara. La Causa-R, MAS, y Convergencia comenzaron el período de 1994-1999 con delegaciones numerosas por primera vez ${ }^{4}$. El número exacto de escaños de cada partido de la Cámara de Diputados varió porque el Consejo Supremo Electoral tomaba decisiones sobre las disputas entre partidos y porque los diputados se cambiaban de partidos (Subero 1997). La división de La Causa-R y la creación del partido parlamentario Independientes por Venezuela significó que a mediados de 1997 siete partidos importantes estaban representados en la Cámara.

Los partidos que tuvieron delegaciones numerosas en el congreso por primera vez se diferenciaron de Acción Democrática y COPEI de manera relevante en la representación. están más descentralizados y menos organizados verticalmente, e imponen menos disciplina sobre sus miembros que Acción Democrática y COPEI. Estas diferencias institucionales crearon un incentivo de comportamiento único para sus miembros. La Causa-R, MAS, Convergencia e Independientes por Venezuela son partidos descentralizados y Acción Democrática y COPEI son centralizadoss.

Si se compara a los diputados de los partidos centralizados y descentralizados, aquellos están más inseguros electoralmente. La estructura partidista de La Causa-R, MAS, Convergencia e Independientes por Venezuela es menos estable que la de Acción Democrática y COPEI. La Causa-R y MAS, por ejemplo, se dividieron en 1997 como resultado de conflictos internos ${ }^{6}$. Así, muchos diputados electos por Convergencia abandonaron su partido durante su término en el Congreso. Dadas sus tendencias hacia el conflicto y la división internos, los diputados de estos partidos no pueden depender de la organización, ni de las redes partidistas para promover sus candidaturas. Como resultado de estas observaciones, los diputados de los partidos descentralizados perciben una mayor inseguridad electoral que los de los centralizados. Cuando se les preguntó quién tenía la responsabilidad de organizar las campañas electorales, el 51,4\% de los diputados de los partidos descentralizados decían que sólo el candidato era responsable, y el 17,1\% dijo que el partido era responsable (Tabla I-A). En contraste, cerca de un cuarto de los diputados de los partidos centralizados dijo que los partidos son los responsables de organizar las campañas electorales, y un tercio indicó que la responsabilidad recaía en los candidatos.

\begin{tabular}{|c|c|c|c|c|c|c|}
\hline \multirow[b]{3}{*}{$\begin{array}{l}\text { Responsibilidad } \\
\text { en las campañas }\end{array}$} & \multicolumn{3}{|c|}{ A. Estructuras partidistas } & \multicolumn{3}{|c|}{ B. Leyes electorales } \\
\hline & Cen. & Descen. & Total & Lista & Circuito & Total \\
\hline & & & & & & \\
\hline Candidato & $34,5 \%$ & $51,4 \%$ & $43,8 \%$ & $22,7 \%$ & $56,1 \%$ & $44,4 \%$ \\
\hline Candidato y partido & $41,4 \%$ & $31,4 \%$ & $35,9 \%$ & $50,0 \%$ & $26,8 \%$ & $34,9 \%$ \\
\hline Partido & $24,1 \%$ & $17,1 \%$ & $20,3 \%$ & $27,3 \%$ & $17,1 \%$ & $20,6 \%$ \\
\hline Total & $100,0 \%$ & $99,9 \%$ & $100,0 \%$ & $100,0 \%$ & $100,0 \%$ & $100,0 \%$ \\
\hline $\mathrm{N}$ & 29 & 35 & 64 & 22 & 41 & 63 \\
\hline()$_{3}^{2}$ & 1,86 & & & $6,51^{*}$ & & \\
\hline
\end{tabular}

Fuente: Kulisheck (1997).

${ }^{*} \mathrm{p}<, 05$.

La inseguridad electoral es un incentivo para que los diputados de partidos descentralizados crean vínculos con intereses que los puedan ayudar políticamente. Sentimientos contra Acción Democrática y COPEI surgidos entre muchos de los grupos emergentes dan a los miembros de los partidos descentralizados una ventaja sobre los de los centralizados, porque los partidos descentralizados comparten preocupaciones programáticas e ideológicas con dichos grupos. La Causa-R, por ejemplo, ha trabajado mucho con los movimientos locales promoviendo intereses emergentes y su éxito electoral se debió en parte a que el partido supo forjar alianzas con grupos e intereses que se oponían a la hegemonía política y social de Acción Democrática y COPEI a niveles, sobre todo, locales y estatales (López Maya 1995).

3. Véase Ramos (1997) para un análisis amplio de los valores y las creencias de los miembros de la Cámara de Diputados del periodo 1994 1999.

4. La Causa-R se separó en dos partidos en marzo de 1997 (Patria Para Todos y La Causa-R). Para este artículo, los dos partidos de La Causa$\mathrm{R}$ se combinarán en un mismo análisis porque la estructura organizativa de estos nuevos partidos no estaba firmemente establecida cuando se produjo la recogida de datos.

5. La codificación de los partidos se determina por 1) el grado en que las reglas de los partidos permiten a los diputados expresar y votar sus consciencias en comités y en la Cámara -en vez de hacer respetar estrictamente una línea partidista-;2) reconocen la legitimidad de facciones internas; 3 ) permiten que los diputados escojan el liderazgo de la delegación en el congreso sin interferencias de la directiva nacional del partido.

6. La división de MAS no afectó la recolección de datos para este proyecto 


\section{Reforma electoral}

La formación de la Comisión Presidencial Para la Reforma del Estado (COPRE) en 1984 marca el comienzo de un proceso de reforma que incluye la adopción de un sistema de leyes electorales mixtas para la Cámara de Diputados. El Presidente Jaime Lusinchi formó el COPRE para satisfacer la petición de algunos segmentos reformistas de la sociedad durante la campaña electoral de 1983. COPRE criticó abiertamente el papel de los partidos políticos y pidió la descentralización del poder político, así como deseó que se autorizara a los electores individuales y a los miembros regulares de los partidos (Veneconomy Montbly 1986: 22-23). COPRE abogó por la elección directa de los gobernadores, la creación de los puestos para alcaldes, la financiación estatal de las campañas electorales, circuitos uninominales para elegir representantes del congreso, asambleas estatales, consejos locales, así como procesos de nominaciones transparentes y más democráticas en los partidos (Gómez Calcaño y López Maya 1990: 120-128).

El Congreso de 1994-1999 fue el primero que usó leyes electorales mixtas. De 1958 a 1988 los venezolanos votaron por listas partidistas cerradas en vez de por candidatos. Bajo este sistema, los diputados enfrentaron incentivos para cultivar vínculos con las élites de los partidos que crearon las listas electorales y establecieron intereses asociados con sus partidos (Carey 1996; Martz 1992). Al usar un sistema proporcional de miembros mixtos, la mitad de la Cámara de Diputados fue elegida de las listas tradicionales de los partidos y la otra mitad de circuitos uninominales por primera vez en 1993.

El debate sobre la adopción de las leyes electorales mixtas implica que los reformistas entendieron las implicaciones potenciales de la representación en las nuevas reglas. Los grupos de la sociedad civil apoyaron, y los partidos tradicionales se opusieron, al componente de los circuitos uninominales en la nueva ley electoral (Véase Ellner 1993; Shugart 1992). Los partidos no quisieron que la sociedad civil esperara que los circuitos uninominales redujeran el control de los partidos en procesos de representación legislativa. Esto hacía que los diputados fueran más responsables porque los partidos apoyaban sus reelecciones. Esta interpretación del impacto de circuitos uninominales surgió del análisis de las experiencias políticas en EE.UU. y Europa (Cain, Ferejohn, y Fiorina 1987; Fenno 1978; Lancaster y Patterson 1990; Mayhew 1974; Weaver y Rockman 1993). Las investigaciones sobre representación en países demostraban que no existían demasiados beneficios electorales de actividades locales en sistemas electorales de representación proporcional, porque los ciudadanos votan por los partidos en vez de por los candidatos directamente. En sistemas de circuitos uninominales, los legisladores no pueden depender de sus conexiones con los partidos para ganar las elecciones. Ellos desarrollan vínculos personales con los intereses locales, porque los ciudadanos pueden usar señales personales, así como las de los partidos cuando están tomando sus decisiones para votar.

Los diputados de circuitos uninominales, que han de competir en elecciones personalizadas en Venezuela, dis- frutan de más independencia de sus partidos que los de las listas partidistas. Una mayoría de los legisladores de los circuitos uninominales exigen que los candidatos sean responsables sólo de las campañas electorales; solamente el 17,1\% defendía que los partidos eran los únicos responsables de la organización de las campañas electorales (Tabla 1-B). Por otra parte, entre los diputados elegidos de las listas partidistas, más de tres cuartos $(50,0+27,3)$ indicaron que los partidos comparten responsabilidades con los candidatos, o que los partidos son completamente responsables de la organización de dichas campañas.

Los diputados de circuitos uninominales tienen incentivos naturales para atender a los intereses locales y estatales porque dependen, en parte, de la reputación en el circuito y de organizaciones personales. Estos incentivos para cultivar vínculos con grupos e intereses locales y estatales pueden ser minados, sin embargo, por la experiencia limitada que puedan tener en la arena política formal. De todas formas, los diputados de circuitos uninominales tienen razones más fuertes para crear vínculos con las organizaciones locales y estatales que sus colegas que ganan la elección basados en su posición en listas de partidos cerradas.

El sistema político venezolano cambió significativamente a principios de los 90. El sistema bipartidista fracasó y los políticos implementaron la ley electoral mixta. Estos cambios eran reformistas, aunque también tenían implicaciones potencialmente radicales si fallaban. Se pensó que las alternativas políticas radicales, como la de Hugo Chávez, estaban inversamente relacionadas con el éxito de las reformas institucionales.

\section{HIPÓTESIS Y ANÁLISIS}

Esta estrategia reformista para mantener la política venezolana se centró en el cambio de sistema partidista y leyes electorales. Se esperaba que las nuevas leyes electorales y la representación política más amplia en el congreso crearan incentivos electorales para que los diputados estuvieran atentos a los intereses emergentes locales y estatales, principalmente. Al conectar sociedad y gobierno, los reformistas pensaron que el apoyo popular de opciones políticas radicales como Hugo Chávez sería minado.

Este artículo adopta el argumento implícitamente hecho por los reformistas venezolanos, y defiende una hipótesis basada en que los diputados de circuitos uninominales y partidos descentralizados atenderán a las demandas de los intereses emergentes porque son más responsables de sus propias reelecciones que sus homónimos de los partidos centralizados. Las estructuras partidistas y las leyes electorales han demostrado ya cómo los diputados perciben las campañas y elecciones (Tabla II). Los diputados de los partidos descentralizados y de los circuitos uninominales no creen que pueden confiar en que las maquinarias partidistas los elijan y, como resultado de ello, creen en su inseguridad electoral. Los diputados de los partidos centralizados y de las listas partidistas tienen más confianza en sus partidos durante las campañas, se sienten menos responsables de sus reelecciones y, por lo tanto, expresan una seguridad electoral 
más fuerte. Si las estructuras partidistas y las reglas electorales afectan los patrones de representación es una pregunta empírica que será respondida en el resto de este artículo. La victoria de Hugo Chávez en 1998 sugiere, sin embargo, que la estrategia reformista por el cambio no satisfizo las expectativas.

Respuestas de diputados sobre representación, presión política
y áreas de destreza en el Congreso

Fuente: Kulisheck (1997)

\section{Análisis 1}

Antes de examinar si las estructuras partidistas y las leyes electorales afectan los patrones de representación, se necesita definir los intereses que tienen acceso al Congreso. Se usan tres variables para medir la representación en la Cámara de Diputados. Primeramente, se preguntó a los diputados directamente qué intereses representaban en el Congreso. Probablemente, las respuestas a esta pregunta estaban muy influidas por la opinión pública, e iban a reflejar más los intereses que los diputados creían que debían representar que los que realmente representaban. Segundo, la encuesta preguntó cuáles son los intereses que presionaban más a los diputados. De manera diferente a la primera variable, la pregunta sobre la presión política mide los contactos reales que existen entre diputados e intereses específicos. Los grupos que mantienen contactos con los diputados están obviamente integrados en la arena legislativa y potencialmente pueden influir en los resultados políticos. Tercero, los diputados declararon sus destrezas en el Congreso. Para promover las demandas de intereses, los diputados necesitan conocer sus asuntos y, por lo tanto, las áreas de destreza de los diputados indican los intereses que reciben atención en el Congreso.

Los intereses geográficos y emergentes ${ }^{7}$ están involucrados en la política legislativa, pero su potencia para influir en los resultados parece limitada. Los diputados perciben que asociarse a intereses geográficos va a ser más beneficiosa políticamente: el 63,9\% indicó que representaba a estos intereses (Tabla II). Sin embargo, los intereses geográficos no mantenían muchos contactos con los diputados para presionarlos, solamente el 10,4\% de los diputados dijo que era presionado por ellos. Unos cuantos más diputados reportaron que tenían destrezas asociadas con estos intereses $(12,5 \%)$. La gran mayoría de los diputados dijo que no repre- sentaba intereses emergentes $(3,3 \%)$, pero más de una quinta parte reportó contactos con representantes de estos intereses $(22,9 \%)$. Aunque los intereses emergentes están presentes en el congreso, pocos diputados expresaron que eran expertos en sus áreas $(6,3 \%)$. Recientemente los partidos nuevos han usado investigaciones para influir en el presidente y en los procedimientos legislativos. Pos eso, el 18,7\% de los diputados indicó que era experto en asuntos relacionado con dichas investigaciones.

Los sindicatos ${ }^{8}$ y la patronal ${ }^{9}$ no son populares entre el pueblo venezolano. Por esta razón, solamente el 3,3\% de los diputados reportó que representaba a los sindicatos, y el $1,6 \%$ expresó que representaban a la patronal en el Congreso (Tabla II). Dada la larga historia de influencias en la políti$\mathrm{ca}$, las respuestas de los diputados indican que temen ser relacionados con intereses tan poco populares. Los sindicatos y la patronal, aún así, mantienen mucha presión sobre los miembros de la Cámara de Diputados. Los sindicatos contactaban con el 45,6\% de los diputados, y los representantes de la patronal tienen contactos con el 14,6\% de los diputados. La patronal hace menos presión a los diputados que los sindicatos, pero el $14,6 \%$ de los diputados respondió que era experto en asuntos financieros o comerciales, y solamente el 8,3\% reportó que sus destrezas estaban relacionadas con los intereses de los sindicatos. Como los sindicatos y la patronal, los partidos son actores tradicionales y no tienen buena fama entre el pueblo venezolano. A pesar de la opinión popular, un cuarto de los diputados $(24,6 \%)$ indicó que representaba a sus partidos en el congreso. Este hecho verifica la influencia que los partidos mantienen en procesos electorales y en carreras legislativas.Casi un tercio de los diputados expresaron que eran expertos en otros asuntos tradicionales como asuntos interiores $(29,2 \%)$ y asuntos exteriores $(4,2 \%)$.

La tabla II indica que una amplia selección de intereses fue representada en la Cámara de Diputados durante el término de 1994-1999. Sin embargo, para ser eficaces representantes de intereses en el Congreso, los diputados tienen que ser activos en las deliberaciones congresistas. Cuando se les preguntó sobre sus niveles de actividad en la Cámara, los diputados presionados por los sindicatos y la patronal pertenecen al $88,9 \%$ de los que mantienen un alto nivel de actividad (Tabla III). Entre los que participan poco, 66,6\% están presionados por intereses emergentes y geográficos. Del hecho de que los diputados más influyentes en el congreso mantienen contactos con los intereses tradicionales (sindicatos y la patronal), surge la idea de que el programa para reformar el sistema puntofijista tuvo un éxito relativo. Los intereses nuevos están representados en la Cámara de Diputados, pero estos intereses mantienen contactos con los

7. La variable “intereses geográficos” se sacó de las respuestas: estados, circuitos, regiones, locales, alcaldes, electores, y el pueblo. La variable "intereses emergentes" se sacó de las respuestas: asociaciones de vecinos, ONGs, colectivos, organizaciones de consumidores, grupos de jubilados, la clase media, y la nueva generación (Kulisheck 1997).

8. La variable "sindicatos" se creó de las respuestas: sindicatos, trabajadores, gremios, y campesinos (Kulisheck 1997).

9. La variable "la patronal" se creó de las respuestas: comerciantes, profesionales, empresarios, y intereses financieros (Kulisheck 1997). 
TABLA III

Actividad de diputados en la Cámara de Diputados analizada por presión política

\begin{tabular}{|lcccc|}
\hline & Actividad alta & Actividad media & Actividad baja & Total \\
\hline Presión política de & & & & \\
Sindicatos & $61,1 \%$ & $42,1 \%$ & $22,2 \%$ & $45,7 \%$ \\
Patronales & $27,8 \%$ & $5,3 \%$ & $11,1 \%$ & $15,2 \%$ \\
Intereses geográficos & $0,0 \%$ & $15,8 \%$ & $22,2 \%$ & $10,9 \%$ \\
Intereses emergentes & $11,1 \%$ & $21,1 \%$ & $44,4 \%$ & $21,7 \%$ \\
Otras & $0,0 \%$ & $15,8 \%$ & $0,0 \%$ & $6,5 \%$ \\
Total & $100,0 \%$ & $100,1 \%$ & $99,9 \%$ & $100,0 \%$ \\
$\mathrm{~N}$ & 18 & 19 & 9 & 46 \\
$0{ }^{2}{ }_{8}$ & $16,06^{*}$ & & & \\
\hline
\end{tabular}

Fuente: Kulisheck (1997).

${ }^{*} \mathrm{p}<, 05$.

diputados menos activos que no pueden promover sus demandas eficazmente. Para minar el apoyo de las alternativas más radicales, los intereses emergentes asociados con la sociedad civil y ubicados al nivel local y estatal necesitaban conseguir una representación substanciosa en la política formal. Todavía es posible que los diputados de los partidos descentralizados y quienes fueron elegidos en circuitos uninominales mantengan vínculos estrechos con los intereses emergentes y geográficos.

\section{Análisis 2}

El fracaso del bipardismo afectó la representación legislativa, sin embargo, la relación entre la centralización de partidos y la representación de intereses emergentes y geográficas no es fuerte estadísticamente (Tabla IV-A). Los datos indican que los diputados de los partidos descentralizados creían que las relaciones con los sindicatos y la patronal beneficiarían más sus carreras que si se asociaban con los intereses emergentes y geográficas. El $62,9 \%(51,8+11,1)$ de los diputados estaban presionados por los representantes de los sindicatos y la patronal. Se esperaba que los diputados de los partidos descentralizados evitaran vínculos con estos intereses en vez de relacionarse con los intereses emergentes al nivel local y estatal porque éstos son autónomos de Acción Democrática y COPEI. Un cuarto de estos diputados expresó que tenía contactos con intereses emergentes $(25,9 \%)$, pero solamente el 3,7\% indicó que estaba presionado por intereses geográficos. En contra de la hipótesis, la inseguridad electoral parece un incentivo para que los diputados sostengan relaciones con los intereses tradicionales.

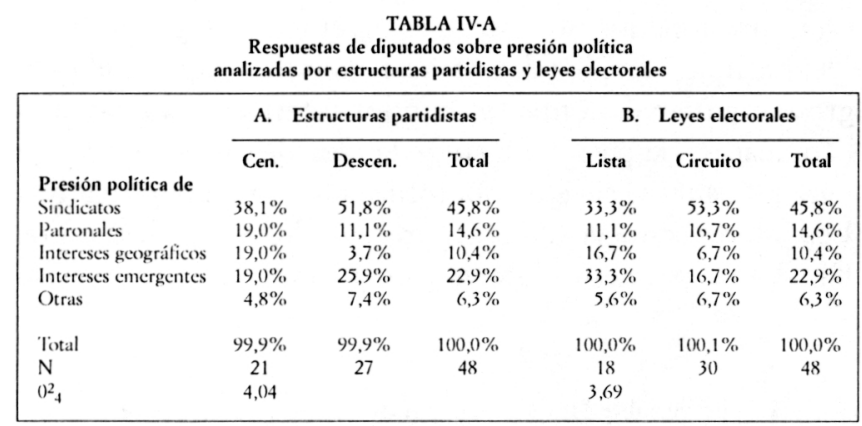

Fuente: Kulisheck (1997).

Los diputados de partidos centralizados mantienen unos vínculos fuertes con una amplia selección de intereses políticos (Tabla IV). Semejante a sus colegas en partidos descentralizados, una pluralidad de los diputados centralizados reportó presión regular de los sindicatos (38,1\%). El 19\% de los diputados de partidos centralizados era presionado por la patronal, intereses emergentes y geográficos. Como resultado de estos datos, parece que estos diputados tienen suficiente libertad para dedicarse a conseguir contactos con intereses diferentes. En contraste con los diputados de partidos descentralizados, los de partidos centralizados creen que sus vínculos partidistas les ayudarán en sus campañas de reelección. Pos eso, más que un tercio de estos diputados $(19+19=38 \%)$ disfruta suficiente seguridad electoral y cree que puede mantener relaciones con intereses nuevos sin dañar su carrera. En términos de estructuras partidistas, la seguridad -en vez de inseguridad-impulsó a los diputados a confrontar los intereses emergentes y geográficos.

Las leyes electorales influyen en la representación como las estructuras partidistas. Los diputados de los nuevos circuitos uninominales estaban especialmente atentos a los representantes de los sindicatos y la patronal, aunque los de listas partidistas mantenían patrones de representación más amplia (Tabla IV-B). Una mayoría de los diputados uninominales indicó que estaba presionada por los sindicatos $(53,3 \%)$. El mismo porcentaje de estos diputados reportó que recibía presión de la patronal y de los intereses emergentes $(16,7 \%)$. Los diputados de circuitos uninominales estaban más atentos a los sindicatos y la patronal que sus colegas elegidos por listas partidistas. Un tercio de los diputados de listas expresó que tenía contactos con los intereses emergentes; y el mismo porcentaje reportó que tenía contactos con los sindicatos. Del mismo modo, un porcentaje más alto de estos diputados tenía más vínculos con intereses geográficos $(16,7 \%)$ que con la patronal $(11,1 \%)$. Como los diputados de los partidos centralizados, los elegidos por listas tienen bastante libertad para dedicarse a diferentes intereses como los nuevos y los tradicionales.

El fracaso del sistema bipartidista y la aplicación de la nueva ley electoral mixta no promovieron patrones de representación como los reformistas predijeron. Los diputados que tuvieron más inseguridad electoral, como resultado de los cambios institucionales, buscaban vínculos con los intereses tradicionales como los sindicatos y la patronal. Es probable que para estos diputados inseguros, los sindicatos y la patronal eran atractivos porque tienen una larga experiencia de actividad en la política formal en Venezuela. De manera diferente a la de los intereses emergentes al nivel local y estatal, los intereses tradicionales tienen los recursos y las redes establecidas para promover candidaturas y carreras políticas.

Los diputados que promovían los asuntos de intereses emergentes y geográficos eran los que tenían más seguridad electoral; principalmente los de partidos centralizados y las listas electorales. Estos diputados, sin embargo, enfrentan las presiones de las élites en sus partidos para observar la línea oficial partidista. Normalmente, sus líneas apoyan los asuntos de los intereses tradicionales. Finalmente, los cambios y reformas tuvieron impactos en el congreso, pero parece que los patrones de representación continúan favoreciendo a los intereses tradicionales, como los sindicatos y la patronal. 


\begin{tabular}{|c|c|c|c|c|}
\hline \multicolumn{5}{|c|}{$\begin{array}{c}\text { APÉNDICE } \\
\text { A.I } \\
\text { Resumen de las codificaciones de la centralización de los partidos }\end{array}$} \\
\hline & \multicolumn{4}{|c|}{ Las estructuras de los partidos permitenal: } \\
\hline & $\begin{array}{c}\text { Facciones } \\
\text { internas }\end{array}$ & $\begin{array}{c}\text { Conducta } \\
\text { independiente }\end{array}$ & $\begin{array}{l}\text { Decisiones } \\
\text { de liderazgo }\end{array}$ & Codificación \\
\hline Partidos & & & & \\
\hline Acción Democrática & No & Poco & No & Centralizado \\
\hline COPEI & No & Poco & No & Centralizado \\
\hline MAS & Sí & $\mathrm{Si}$ & $\mathrm{Si}$ & Descentralizado \\
\hline Convergencia & $\mathrm{Sí}$ & Sí & Si & Descentralizado \\
\hline La Causa-R & No está claro & $\mathrm{Si}$ & $\mathrm{Sí}$ & Descentralizado \\
\hline Indep, Por Venezuela & $\mathrm{Si}$ & $\mathrm{Si}$ & $\mathrm{Si}$ & Descentralizado \\
\hline
\end{tabular}

Fuente: Kulisheck (1998).

a Las codificaciones vienen de un análisis de los Reglamentos y/o Estatutos de los partidos. El análisis determinó si las reglas y estructuras de los partidos (1) reconocen la legitimidad de facciones internas; (2) determinó si las reglas y estructuras de los partidos (1) reconocen la legitimidad de facciones internas; (2)
permiten que los diputados se comporten independientemente de la línea partidista, si permiten el voto permiten que los diputados se comporten independientemente de la línea partidista, si permiten el voto
de conciencia; y (3) permiten que los diputados escojan el liderazgo de la delegación en el Congreso sin de conciencia; y (3) permiten que los diputados esco
interferencias de la directiva nacional del partido.

\section{CONCLUSIONES. LA ELECCIÓN DE 1998}

Hugo Chávez no ganó la presidencia como resultado del impacto limitado del fracaso del sistema bipartidista y la adopción de una ley electoral mixta. Un pasado de reformas con efectos marginales, sin embargo, creó condiciones que favorecieron a los movimientos políticos que rechazaban las propuestas de cambios moderados. En 1998, la mayoría de los ciudadanos vio la reforma electoral y los cambios al sistema partidista como ejemplos más recientes de programas reformistas que habían fallado en el gobierno y la sociedad civil.

Así, la elección de 1998 fue una elección entre vagas promesas de cambios radicales e implícitos respaldos a una continuación de la agenda reformista. Hugo Chávez y su partido el Movimiento V República declararon la necesidad de una ruptura radical con el pasado en Venezuela. La complicidad de Chávez en el intento de golpe de estado en febrero de 1992 hizo creíbles sus promesas para gran parte de la población, particularmente para la gente pobre en los barrios urbanos. Sus retos generales eran siempre claros: parar la corrupción, debilitar los partidos tradicionales y dar poder al pueblo venezolano. La manera de realizarlos estaba mucho menos clara durante la campaña. Convocó la Asamblea Constituyente para reescribir la Constitución del 61, pero nunca especificó qué actores y reglas reeplazarían a los partidos actuales y a las instituciones de la presidencia y el congreso. El análisis de los resultados electorales del 98 indica que los venezolanos decidieron que las propuestas obscuras para un cambio radical de Chávez prometían más que la continuación de pequeñas reformas del pasado.

El radicalismo fue aceptable porque el movimiento reformista había fracasado a la hora de confrontar la crisis política y económica de Venezuela que había durado una década. Los otros dos candidatos importantes de la campaña de 1998, Henrique Salas Römer e Irene Sáez, estaban asociados con este movimiento reformista. Salas Römer fue gobernador del estado de Carabobo durante dos períodos al principio de los 90. Su administración se identificaba como una de las más exitosas desde la creación del puesto en 1989. Como gobernador, adoptó programas creativos de empleo y pidió inversiones domésticas e internacionales para su estado. Carabobo fue un atractivo para inversionistas gracias a este gobierno tan eficaz. Salas Römer ha sido una constante voz promotora de la descentralización del poder político y económico del gobierno nacional hacia los estados y municipios. De igual manera, Salas Römer ha apoyado fuertemente la expansión del uso de circuitos uninominales en elecciones legislativas en todos los niveles del gobierno. La campaña de Sala Römer enfatizó los temas de descentralización y reforma electoral y, con menos fuerza, el mantenimiento de la agenda económica neo-liberal empezada por el gobierno de Caldera.

La segunda candidata reformista fue Irene Sáez, la alcaldesa del municipio Chacao. Ella ganó la reelección en este suburbio rico de Caracas en 1995 con aproximadamente el $96 \%$ del voto. Estableció vínculos con grupos locales y con muchas organizaciones de la sociedad civil. Hasta que fue candidata para la presidencia, Sáez se habían separado de los partidos políticos. De hecho, su aceptación al nombramiento oficial de COPEI contribuyó a su caída en las encuestas de la opinión pública de $1998^{10}$. En Chacao se le consideraba una alcaldesa eficaz y honesta que había respondido a las preocupaciones de los residentes de su municipio. Fue particularmente popular por su éxito en programas de limpieza del municipio y mejoramiento de los servicios policiales y del sistema de ambulancias. El argumento implícito en la campaña presidencial de Sáez fue que ella sería capaz de extender su programa político de Chacao a toda Venezuela.

Salas Römer y Sáez crearon sus carreras políticas en puestos asociados estrechamente con la agenda reformista. Si las reformas hubieran sido evaluadas siguiendo sólo estos dos ejemplos, habrían tenido gran éxito. Pero no todos los estados y municipios habían sido gobernados con tanta eficacia como Carabobo y Chacao. Además, los reformistas no habían confrontado los problemas sistemáticos a nivel nacional que son los que realmente determinan si los ciudadanos tienen un empleo que les permita realizar una vida de clase media como era la norma durante la época petrolera. Por eso, la alternativa reformista no fue muy popular entre la gran mayoría de los venezolanos en 1998.

Cuando votaron, los venezolanos creyeron que el cambio radical ofrecía la única posibilidad para que el país volviera a un pasado rico y estable. El gobierno de Chávez podría enfrentar unas expectativas demasiado altas si los ciudadanos se basaban en sus recuerdos del 70 y de las ganancias del petróleo. Debido a que el modelo de representación partidista del sistema Punto Fijo ha sido rechazado y la agenda reformista ha sido puesta en descrédito por una mayoría, será incierta la forma que tomará la próxima etapa en la evolución de la democracia venezolana, si el gobierno de Chávez no cumple con sus promesas políticas.

10 En las últimas dos semanas de la campaña, COPEI revocó el nombramiento de Sáez y apoyó a Salas Römer. Acción Democrática hizo lo mismo y revocó su nombramiento de Luis Alfaro Ucero para endorsar la candidatura de Salas Römer. 


\section{BIBLIOGRAFÍA}

Alvarez, Angel E., ed. (1996a): El Sistema Político Venezolano: Crisis y Transformaciones. Caracas: Universidad Central de Venezuela.

AlvareZ, Angel E. (1996b): "La crisis de hegemonía de los partidos políticos venezolanos.” En A. Alvarez, ed., El Sistema Político Venezolano: Crisis y Transformaciones, pp. 131-154. Caracas: Universidad Central de Venezuela.

Cain, Bruce E., John A. Ferejohn y Morris P. Fiorina (1987): The Personal Vote: Constituency Service and Electoral Independence. Cambridge, MA: Harvard University Press.

CANACHE, Damarys J. y Michael R. KulISHECK, eds. (1998): Reinventing Legitimacy: Democracy and Political Change in Venezuela. Westport, CT: Greenwood.

CareY, John M. (1996): Term Limits and Legislative Representation. New York: Cambridge University Press.

Coppedge, Michael (1994): Strong Parties and Lame Ducks. Presidential Partyarchy and Factionalism in Venezuela. Stanford, CA: Stanford University Press.

CrISP, Brian F. y Daniel H. Levine (1998): "Democratizing the Democracy? Crisis and Reform in Venezuela." Journal of Interamerican Studies and World Affairs 40: 27-61.

ELLnER, Steve (1993): "The Deepening of Democracy in a Crisis Setting: Political Reform and the Electoral Process in Venezuela." Journal of Interamerican Studies and World Affairs 35: 1-42.

FEnNo, Richard R. Jr. (1978): Home Style: House Members in Their Districts. New York: Harper-Collins.

García-Guadilla, María Pilar (1992): "The Venezuelan Ecology Movement: Symbolic Effectiveness, Social Practices, and Political Strategies." En A. Escobar y S. Alvarez, eds. The Making of Social Movements in Latin America: Identity, Strategy, and Democracy, pp. 150-170. Boulder, CO: Westview Press.

GÓMEZ CALCAÑO, Luis (1998): "Civic Organization and the Reconstruction of Democratic Legitimacy in Venezuela". En D. Canache y M. Kulisheck, eds., Reinventing Legitimacy: Democracy and Political Change in Venezuela, pp. 169-185. Westport, CT: Greenwood Press.

Gómez CalCaÑo, Luis y Margarita López MAYA (1990): El Tejido de Penélope: La Reforma del Estado en Venezuela (1984-1988). Caracas: CENDES/APUCV-IPP.

GoOdMAn, Louis W., Johanna MENDELSON Forman, Moisés Naím, Joseph S. Tulchin y Gary Bland, eds. (1995): Lessons of the Venezuelan Experience. Washington, D.C. y Baltimore: The Woodrow Wilson Center Press y The Johns Hopkins University Press.

Guerón, Gabrielle y Giorgio MANCHISI (1996): "La descentralización en Venezuela: Balance de un proceso inconcluso". En A. Alvarez, ed., El Sistemo Político Venezolano: Crisis y Transformaciones, pp. 353-406. Caracas: IEP-UCV.

Hellinger, Daniel (1996): "The Causa-R and the Nuevo Sindicalismo in Venezuela." Latin American Perspectives 23: 110-131.
HochsteTleR, Kathryn (1997): "The Evolution of the Brazilian Environmental Movement and Its Political Roles." En D. Chalmers, et al., eds., The New Politics of Inequality in Latin America, pp. 192-216. London: Oxford University Press.

KarL, Terry Lynn (1986): "Petroleum and Political Pacts: The Transition to Democracy in Venezuela." En G. O'Donnell, P. Schmitter y L. Whitehead, eds., Transitions from Authoritarian Rule: Latin America, pp. 196-219. Baltimore: The Johns Hopkins University Press

KARL, Terry Lynn (1995): "The Venezuelan Petro-State and the Crisis of 'Its' Democracy." En J. McCoy, A. Serbin, W. Smith, y A. Stambouli, eds., Venezuelan Democracy Under Stress, pp. 33-55. New Brunswick, NJ: Transaction Publishers.

KarL, Terry Lynn (1997): The Paradox of Plenty: Oil Booms and Petro-States. Berkeley, CA: University of California Press.

Kornblith, Miriam y Daniel H. Levine (1995): "Venezuela: The Life and Times of the Party System." En S. Mainwaring y T. Scully, eds., Building Democratic Institutions: Party Systems in Latin America, pp. 37-71. Stanford, CA: Stanford University Press.

KULISHECK, Michael R. (1997): University of Pittsburgh Legislator Opinion Project. Interview data from the Venezuelan Chamber of Deputies, March-June 1997.

KULISHECK, Michael R. (1998): Legislators, Representation, and Democracy: An Institutional Analysis of Deputy Responsiveness in Venezuela. Ph.D. diss., University of Pittsburgh, Pittsburgh.

Kulisheck, Michael R. y Damarys J. Canache (1998): "Democratic Legitimacy and Political Change in Venezuela." En D. Canache y M. Kulisheck, eds., Reinventing Legitimacy: Democracy and Political Change in Venezuela, pp. 39-50. Westport, CT: Greenwood Press.

LANCASTER, Thomas D. y W. David PATTERSON (1990): "Comparative Pork Barrel Politics: Perceptions of the West German Bundestag" Comparative Political Studies 22: 458-477.

Levine, Daniel H. (1994): "Goodbye to Venezuelan Exceptionalism." Journal of Interamerican Studies and World Affairs 36: 145-182.

Levine, Daniel H. (1998): "Beyond the Exhaustion of the Model: Survival and Transformation of Democracy in Venezuela." En D. Canache y M. Kulisheck, eds., Reinventing Legitimacy: Democracy and Political Change in Venezuela, pp. 187-214. Westport, CT: Greenwood Press.

Levine, Daniel H. y Brian F. CRISP (1995): "Legitimacy, Governability, and Reform in Venezuela." En L. Goodman, J. Mendelson Forman, M. Naím, J. Tulchin, y G. Bland, eds., Lessons of the Venezuelan Experience, pp. 223-251. Washington, D.C. y Baltimore: The Woodrow Wilson Center Press y The Johns Hopkins University Press.

LÓPEz MAYA, Margarita (1995): "El ascenso en Venezuela de la Causa R." Revista Venezolana de Economía y Ciencias Sociales 2-3: 57-84. 
LóPEz Maya, Margarita (1998): "New Avenues for Popular Representation in Venezuela: La Causa- $R$ and the Movimiento Bolivariano 200.” En D. Canache y M. Kulisheck, eds., Reinventing Legitimacy: Democracy and Political Change in Venezuela, pp. 83-95. Westport, CT: Greenwood Press.

MARTZ, John D. (1992): "Party Elites and Leadership in Colombia and Venezuela." Journal of Latin American Studies 24: 87-121.

MARTZ, John D. (1998): "Deconstruction versus Reconstruction: The Challenge to Venezuelan Parties." En D. Canache y M. Kulisheck, eds., Reinventing Legitimacy: Democracy and Political Change in Venezuela, pp. 65-81. Westport, CT: Greenwood Press.

MAYHew, David R. (1974): Congress: The Electoral Connection. New Haven: Yale University Press.

McCoy, Jennifer, Andrés Serbin, William C. Smith, and Andrés Stambouli, eds. (1995): Venezuelan Democracy Under Stress. New Brunswick, NJ: Transaction Publishers.

RAmOS, María Luisa (1997): "Creencias y valores de los parlamentarios en Venezuela." Nueva Sociedad 148 (MarzoAbril): 44-50.

Rey, Juan Carlos (1972): "El Sistema de Partidos Venezolano". Politeia 1: 175-230.

Rey, Juan Carlos (1994): "Polarización Electoral, Economía del Voto y Voto Castigo en Venezuela: 1958-1988." Cuestiones Políticas 12: 3-96.
SalamanCA, Luis (1995): "The Venezuelan Political System: A View from Civil Society." En J. McCoy, A. Serbin, W. Smith, y A. Stambouli, eds., Venezuelan Democracy Under Stress, pp. 197-213. New Brunswick, NJ: Transaction Publishers.

SHUGART, Matthew Soberg (1992): "Leaders, Rank and File, and Constituents: Electoral Reform in Colombia and Venezuela." Electoral Studies 11: 21-45

Subero, Carlos (1997): "El 9\% de los parlamentarios ha cambiado de fracción.” El Universal (February 23): $1-16$.

Templeton, Andrew (1995): "The Evolution of Public Opinion." En L. Goodman, J. Mendelson Forman, M. Naím, J. Tulchin, y G. Bland, eds., Lessons of the Venezuelan Experience, pp. 79-114. Washington, D.C. y Baltimore: The Woodrow Wilson Center Press y The Johns Hopkins University Press.

Urbaneja, Diego Bautista (1995): Pueblo y petróleo en la politica venezolana del siglo XX. Caracas: Monte Avilia Editores Latinoamericana.

Veneconomy Monthly (1986): May: 22-23.

WEAVER, R. Kent y Bert A. RockMAN (1993): "Assessing the Effects of Institutions.” En R. K. Weaver y B. Rockman, eds., Do Institutions Matter? Government Capabilities in the United States and Abroad, pp. 1-41. Washington, D.C.: The Brookings Institution.

\section{RESUMEN}

En este artículo se analiza la representación en la Cámara de Diputados de Venezuela durante el período de 1994-1999 para contextualizar la candidatura presidencial de Hugo Chávez. En 1993, el sistema bipartidista en Venezuela fracasó y se usó una nueva ley electoral mixta por primera vez. Estas reformas reforzaron la influencia congresista de la patronal y los sindicatos, en vez de ampliar los patrones de representación para incluir las preocupaciones de los intereses emergentes.

El fracaso de las reformas moderadas para mejorar la representación favoreció a candidaturas de políticas que apoyaron las agendas políticas radicales, como la de Hugo Chávez.

Palabras clave: Pacto de Punto Fijo, Cámara de Diputados Venezolana, Reforma Electoral, representación política, Sociedad Civil, Hugo Chávez.

\section{ABSTRACT}

This article examines representation in the 1994-1999 Venezuelan Chamber of Deputies to contextualize the presidencial candidacy of Hugo Chávez. In 1993, the Venezuelan the two-party system broke down and a new mixedmember electoral law for Congress was implemented. These reforms reinforced the congressional influence of business and labor rather than broaden representation patterns to include the concerns of emerging interests. The failure of moderate reforms to improve representation created conditions that favored the candidacies of politicians, like Hugo Chávez, who endorsed radical political agendas.

Key words: Pacto de Punto Fijo, Venezuelan Chambers of Deputies, Electoral Reform, Politic representation, Civil Society, Hugo Chávez. 